\title{
A Conceptual Model of Biopsychosocial Mechanisms of Transition from Acute to Chronic Postsurgical Pain in Children and Adolescents
}

This article was published in the following Dove Press journal:

Journal of Pain Research

\author{
Jennifer A Rabbitts $\mathbb{D}^{1,2}$ \\ Tonya M Palermo (D) ${ }^{1,3,4}$ \\ Emily A Lang ${ }^{5}$
}

'Department of Anesthesiology \& Pain Medicine, University of Washington, Seattle, WA, USA; ${ }^{2}$ Center for Clinical and Translational Research, Seattle Children's Hospital, Seattle, WA, USA;

${ }^{3}$ Center for Child Health, Behavior and Development, Seattle Children's Hospital, Seattle, WA, USA; ${ }^{4}$ Department of Pediatrics, University of Washington, Seattle, WA, USA; ${ }^{5}$ Weill Cornell Medicine, New York, NY, USA
Correspondence: Jennifer A Rabbitts Seattle Children's Hospital, 4800 Sand Point Way NE MB.II.500.3, Seattle, WA 98105, USA

$\mathrm{Tel}+\mathrm{I} 206-987-2704$

Fax + I 206-987-3935

Email jennifer.rabbitts@seattlechildrens. org

\begin{abstract}
Acute and chronic pain are highly prevalent and impactful consequences of surgery across the lifespan, yet a comprehensive conceptual model encompassing biopsychosocial factors underlying acute to chronic pain transition is lacking, particularly in youth. Building on prior chronic postsurgical pain models, we propose a new conceptual model of biopsychosocial mechanisms of transition from acute to chronic postsurgical pain. This review aims to summarize existing research examining key factors underlying acute to chronic postsurgical pain transition in order to guide prevention and intervention efforts aimed at addressing this health issue in children. As pain transitions from acute nociceptive pain to chronic pain, changes in the peripheral and central nervous system contribute to the chronification of pain after surgery. These changes include alterations in sensory pain processing and psychosocial processes (psychological, behavioral, and social components), which promote the development of chronic pain. Patient-related premorbid factors (eg, demographic factors, genetic profile, and medical factors such as premorbid pain) may further modulate these changes. Factors related to acute injury and recovery (eg, surgical and treatment factors), as well as biological response to surgery (eg, epigenetic, inflammatory, and endocrine factors), may also influence this process. Overall, longitudinal studies examining temporal pathways of biopsychosocial processes including both risk and resiliency factors will be essential to identify the mechanisms involved in the transition from acute to chronic pain. Research is also needed to unravel connections between the acute pain experience, opioid exposure, and sensory pain processing during acute to chronic pain transition. Furthermore, future studies should include larger and more diverse samples to more fully explore risk factors in a broader range of pediatric surgeries. The use of conceptual models to guide intervention approaches targeting mechanisms of transition from acute to chronic pain will significantly advance this field and improve outcomes for children and adolescents undergoing surgery.
\end{abstract}

Keywords: chronic postoperative pain, CPSP, neurobiological, risk factors, resilience, quantitative sensory testing

\section{Introduction}

Acute and chronic pain are highly prevalent and impactful consequences of surgery. In pediatric populations, about half of children admitted after surgery experience moderate to severe pain in the hospital, ${ }^{1}$ with rates ranging from $54 \%$ to $91 \%$ following invasive surgeries (eg, spinal fusion, pectus repair). ${ }^{2,3}$ Continued pain at home during the subacute period has been shown to significantly affect recovery. ${ }^{4}$ In a wide range of inpatient surgeries, moderate to severe postsurgical pain at one 
month was independently associated with greater than three-fold higher odds of experiencing a clinically significant deterioration in both physical and psychosocial health, as well as in overall health-related quality of life after surgery. ${ }^{5}$

When acute pain persists after surgery, it can develop into a chronic pain condition. Chronic postsurgical pain (CPSP) is defined as pain localized to the surgical field, without other attributable causes, that continues for more than three months after surgery and impacts health-related quality of life. ${ }^{6,7}$ The majority of studies in the adult and pediatric literature focus only on the presence of pain three or more months after surgery, with rates ranging from $3 \%$ to $15 \%$ after outpatient pediatric surgeries ${ }^{8}$ and $11 \%$ to $35 \%$ after major pediatric surgeries (eg, orthopedic, thoracic) requiring inpatient stays. ${ }^{9,10}$ Unfortunately, CPSP is not only common, but also associated with negative longterm consequences including functional disability and activity limitations, ${ }^{8,10-13}$ as well as low physical and psychosocial health, ${ }^{13}$ and has potential to generate substantial economic costs to healthcare organizations and individual patients. ${ }^{14,15}$ CPSP, when accordingly defined as persistent pain that impacts health-related quality of life, has predominantly been examined after major musculoskeletal surgery and is estimated to occur in around $20 \%$ of these youth. ${ }^{16}$ With the recent expansion of literature focused on this condition, an increasing number of biopsychosocial risk factors have been identified as associated with CPSP in children and adolescents. However, the lack of a conceptual model encompassing these factors hinders our progress in identifying intervention targets and subgroups of patients in which to intervene in order to address this major health issue in children.

The aims of this review are therefore: 1) to present a conceptual model of biopsychosocial mechanisms of transition from acute to chronic postsurgical pain; 2) to summarize the evidence base for key factors (premorbid, injury-related, psychosocial, sensory, and biological factors) in the model that predict either incidence or severity of CPSP in children and adolescents; and 3) to identify gaps and opportunities for further investigations of the transition from acute to chronic postsurgical pain in children that can inform prevention and intervention strategies.

Katz and Seltzer introduced a model of processes involved in the development of CPSP and pain disability in adults, highlighting premorbid patient-related factors (age, sex, pain), surgical factors (surgery duration, surgery approach), and psychological and social factors (catastrophizing, anxiety, social support), as well as the role of genetics, inflammation, and nerve damage. ${ }^{17}$ More recently, Elman and Borsook took a broader approach, drawing from addiction neurobiology research to present a model of evolution from tissue or nerve injury (inciting event), as detected by nociceptors (acute pain), ultimately transitioning to chronic pain. This model highlights contributory processes in the peripheral and central nervous system, including sensory, psychological, behavioral, and social components. ${ }^{18}$ While no pediatric model has been proposed for acute to chronic postsurgical pain transition, pediatric models of chronic pain have described processes promoting adjustment to or maintenance of chronic pain and disability. For example, the fear avoidance model applied to pediatric pain ${ }^{19}$ highlights a pathway of pain catastrophizing, pain-related fear, and avoidance leading to functional disability and maintenance of pain. However, limited focus has been given to precipitating factors that confer risk for development of pediatric pain. Building on the framework of acute to chronic pain transition proposed by Elman and Borsook, ${ }^{18}$ we incorporate perioperative factors from the model of CPSP developed by Katz and Seltzer ${ }^{17}$ to propose a new conceptual model of biopsychosocial mechanisms of transition from acute to chronic postsurgical pain, highlighting factors relevant in children and adolescents.

A Conceptual Model of Biopsychosocial Mechanisms of Transition from Acute to Chronic Postsurgical Pain (Figure 1).

As pain transitions from acute nociceptive pain following surgery to chronic pain and associated impairment in health-related quality of life (CPSP), changes in the peripheral and central nervous system contribute to the chronification of pain. ${ }^{18}$ Alterations in pain processing may lead to amplification of the sensory component of pain, and alterations in psychological, behavioral, and social components may promote evolution of chronic pain. In children, salient psychological, behavioral, and social components include emotional distress (eg, mood and affect), cognitions (eg, fear of pain, catastrophizing), behaviors (eg, sleep), and parent and family factors (eg, family functioning). Patient-related premorbid factors, such as age, sex, genetic profile, and medical factors (eg, premorbid pain), may modulate changes in sensory and psychosocial processes in response to the acute injury. After the inciting event, factors related to the acute injury (eg, the surgical approach) and recovery (eg, treatment factors) influence 


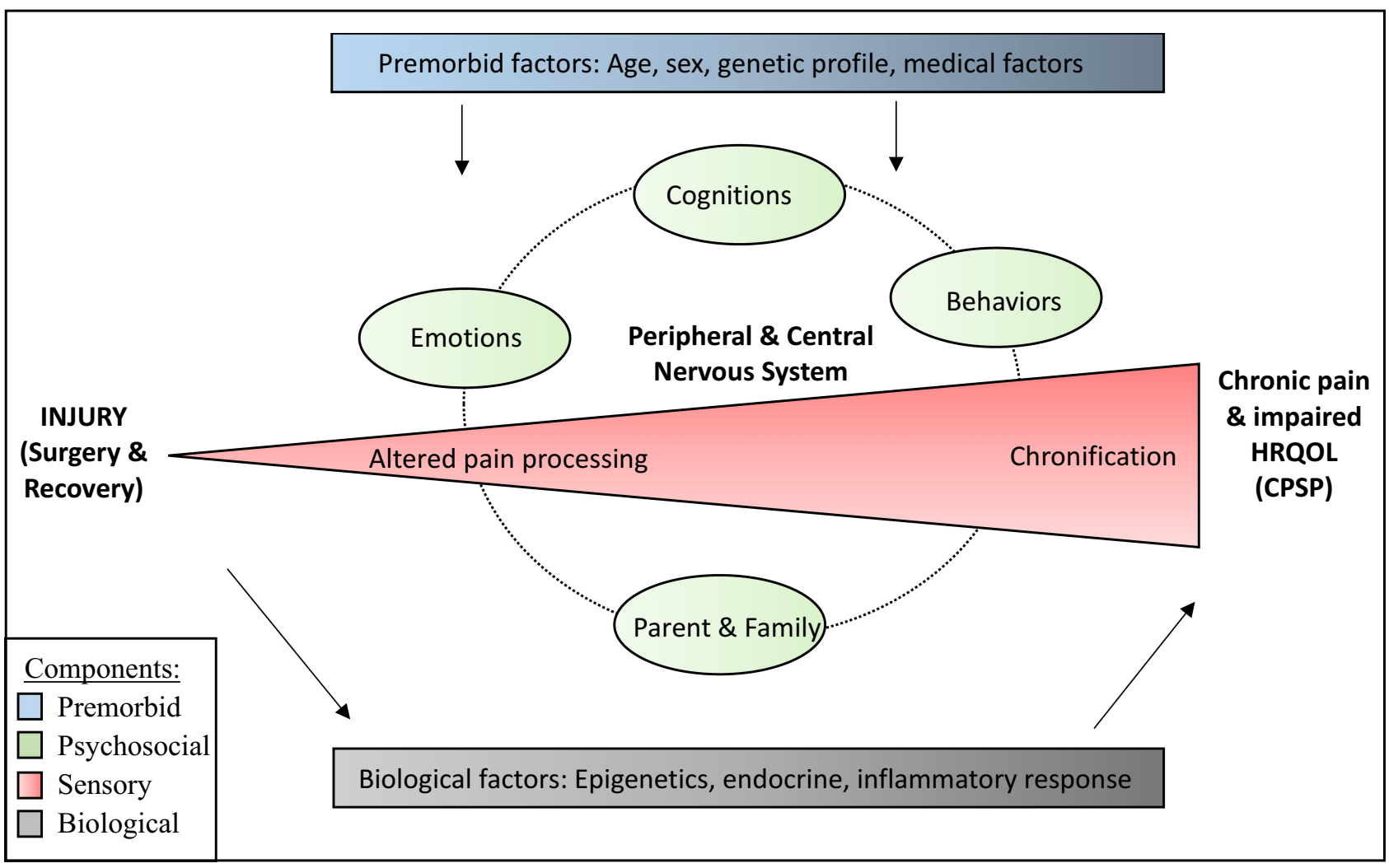

Figure I Conceptual model of biopsychosocial mechanisms of transition from acute to chronic postsurgical pain.

this process. Further, the biological response to surgery, including changes in epigenetic, endocrine, and inflammatory factors, may play an important role in resultant peripheral and central nervous system adaptations.

\section{Premorbid Factors Demographic Factors}

Demographic factors, including the child's age, sex, race and ethnicity, and socioeconomic status, may affect the child's experience of postsurgical recovery and pain. Several studies have found that older child age $\mathrm{e}^{20,21}$ and female $\operatorname{sex}^{3}$ are associated with higher acute pain and pain unpleasantness, as well as with poorer health-related quality of life ${ }^{5,16}$ after surgery. Pediatric chronic pain research in non-surgical samples similarly suggests that older age and female sex are related to higher chronic pain severity and impact. ${ }^{22-25}$ Yet, across pediatric perioperative studies, no associations have been found between pediatric CPSP and child age, ${ }^{13,26,27}$ sex, ${ }^{13,26,28}$ or socioeconomic factors (eg, family income ${ }^{13,26}$ and parents' education). ${ }^{16}$ However, small sample sizes with wide age ranges, as well as limited diversity within samples (eg, inclusion of predominantly white, high-income families) limit our understanding of sociodemographic predictors of CPSP. The inclusion of larger and more diverse samples may expose potential relationships between sociodemographic factors and CPSP.

\section{Genetic Factors}

Emerging research implicates genetic mechanisms in modulating susceptibility to CPSP. Potential genetic mechanisms include both structural DNA sequence variations (single-nucleotide polymorphisms [SNPs]), which can be considered premorbid genetic factors, and changes in DNA function (ie, epigenetic factors such as DNA methylation), which may occur before surgery or as part of the biological response to surgical injury/pain. ${ }^{29}$ A large body of adult perioperative literature points to the role of the catechol-O-methyltransferase (COMT) gene in modulating pain sensitivity and the incidence of chronic pain. ${ }^{30-34}$ One pediatric study found significant relationships between three COMT SNPs and acute postsurgical pain intensity, ${ }^{35}$ suggesting a potential role in interindividual variation in postoperative pain perception. Two studies investigated the association between DNA methylation in 
pain-related genomic pathways, measured before surgery, and the incidence of chronic pain after spinal fusion surgery in adolescents. In one study, increased methylation at the promotor for the mu-1 opioid receptor (OPRMI) gene was associated with persistent pain at two to three months postoperatively. ${ }^{36}$ In addition, utilizing a global bioinformatics-based approach to compare DNA methylation profiles, this same research group found that enrichment of differentially DNA methylated positions in GABA receptor and dopamine pathways related to pain was associated with CPSP. ${ }^{37}$ Notably, enrichment of these DNA methylation profiles was also associated with greater anxiety sensitivity, a psychosocial factor related to $\mathrm{CPSP}^{38}$ highlighting that further research is needed to disentangle these complex interrelationships. Further reasearch in adults has also identified relationships between KCNS1 gene variants in the potassium channel family and chronic postoperative pain. ${ }^{33,39-42}$ Future studies should seek to expand on this adult literature to identify genetic risk factors specific to the development of pediatric CPSP.

\section{Medical Factors}

Pre-existing medical factors, such as preoperative pain and medical history, influence acute and chronic postsurgical pain. The most studied premorbid factor is the presence and severity of preoperative pain, which consistently predicts the severity of acute postoperative pain. ${ }^{16,43,44}$ However, studies focused on the transition to chronic postoperative pain are less clear and point to pain measurement issues. The majority of studies that measured preoperative pain at a single timepoint or based on children's retrospective recall found that intensity of bodily pain before surgery was associated with the incidence of CPSP. ${ }^{9,12,26,27,45,46}$ However, studies that better captured the preoperative pain experience through prospectively recorded daily pain scores over multiple days preoperatively found no relationship with development of CPSP. ${ }^{13,16}$ Several studies have also examined preoperative pain distribution and centralized pain symptoms (fibromyalgia symptoms), dimensions of pain that have garnered a lot of attention in adult surgical populations, ${ }^{47-50}$ as risk factors for pediatric CPSP. Children's fibromyalgia symptoms (ie, greater pain locations and symptom severity before surgery) predicted more severe acute and chronic postsurgical pain when examined as part of a "high symptom cluster". ${ }^{51,52}$ However, when considered as an independent factor, number of preoperative pain locations was not associated with subsequent acute or chronic postoperative pain. ${ }^{16}$ The discrepancies between these results emphasize the need for greater rigor and consistency in the measurement of preoperative pain and suggest that a comprehensive assessment of pain characteristics, including not only pain intensity but also pain distribution and location, pain quality, and pain sensitivity, should be further explored.

Several studies have examined the association between medical comorbidities, chronic conditions unrelated to the planned surgery, prior chemotherapy use, and prior surgery on the incidence of CPSP, ${ }^{9,53}$ finding weak associations. Similarly, no relationship has been found between children's preoperative weight/body mass index and subsequent development of CPSP. ${ }^{8,26,27,46,53}$ However, given the limited investigation of the role of medical factors on the development of CPSP, this is an area that requires further study before conclusions can be drawn.

\section{Injury and Recovery} Factors Related to Injury (Surgery)

A large body of adult literature supports the association between factors related to the surgery itself, such as surgical type and duration, and the incidence of CPSP, potentially due to nerve injury and the subsequent inflammatory response. ${ }^{32}$ However, pediatric studies generally fail to find this relationship. Given that the bulk of pediatric literature focuses on select orthopedic surgeries, it is possible that there has not been enough variation in surgical factors to discern a relationship with CPSP. Several studies examining the incidence of CPSP following major pediatric surgery found that the type of surgery, and emergency versus planned surgery, did not predict CPSP. ${ }^{9,10,13,28}$ Studies focused on spinal fusion surgery also largely failed to identify relationships between surgical factors and pain outcomes: surgical approach, curve parameters (eg, Cobb angle, curve flexibility, shoulder height difference), and indicators of the extent of surgery (spinal fusion length, lowest instrumented vertebrae, scar size, and percent of spinal curve corrected) were not associated with CPSP. ${ }^{9,12,26,27,45,51,53}$ Studies examining surgical duration as a proxy for extent of spine surgery in youth also have not identified a relationship with CPSP. ${ }^{26,51}$ Larger studies are needed in pediatric populations to more fully explore associations between surgical factors and CPSP in a broader range of surgeries. 


\section{Factors Related to Acute Postoperative \\ Recovery}

The acute phase of postoperative recovery is a period encompassing physiological changes, rapid symptom recovery, and acute pain treatment, all of which may impact long-term postsurgical outcomes. Three studies using nuanced approaches to characterize the acute pain trajectory over multiple postoperative days found that higher levels of pain in the hospital and during the initial days at home were associated with a higher incidence of CPSP. ${ }^{4,38,44}$ Acute pain treatment may impact acute recovery, and is integrally related to the acute pain experience. However, studies examining in-hospital pain treatment have not identified a relationship with subsequent CPSP. For example, cumulative in-hospital opioid consumption was not related to the incidence of CPSP after spinal fusion surgery. ${ }^{26,54}$ Similarly, use of regional anesthesia, including neuraxial bocks (eg, epidural, intrathecal/spinal) for postoperative pain control, was not associated with the odds of developing CPSP in observational studies of children undergoing major surgery. ${ }^{9,10,51}$

Looking beyond hospital discharge, studies examining recovery during the initial weeks at home identified significant relationships between subacute pain with subsequent chronic pain. ${ }^{4,16,28}$ In general, studies have relied on self-report measures and data from the electronic medical record to assess acute recovery. Future studies should apply more granular monitoring (such as daily pain diaries) and employ objective measures (eg, actigraphy monitoring of activity, electronic medication monitoring of opioid use) to better capture acute recovery. ${ }^{4}$ Such studies may help to unravel the connections between the acute pain experience, pain treatment, and opioid exposure that play a role in acute to chronic pain transition. Further, mechanistic studies will be essential in understanding physiological perturbations tied to acute recovery that may contribute to the transition from acute to chronic postsurgical pain.

\section{Psychosocial Factors}

\section{Emotional and Cognitive Factors}

A growing body of literature examining the influence of children's psychological health on the development of CPSP has begun to shed light on cognitive, emotional, and behavioral factors that may confer risk for persistent pain. Both general and pain-specific psychological factors have been implicated in pain persistence. ${ }^{55}$ Data exploring general psychological constructs of emotional distress (anxiety, depression) and symptoms of post-traumatic stress disorder have yielded mixed findings. Three studies found that higher baseline anxiety levels before surgery and in the hospital after surgery predicted risk for development and maintenance of CPSP, ${ }^{26,28,38}$ and one more recent study demonstrated that higher depressive symptoms in the week before surgery predicted CPSP in youth undergoing major musculoskeletal surgical procedures. ${ }^{16}$ However, other studies did not identify relationships between these constructs and CPSP. ${ }^{10,11}$

Studies of pain-specific psychological factors include presurgery or immediate postsurgery pain anxiety, pain catastrophizing, pain-related fear, and pain vigilance, as well as protective (resiliency) factors such as pain coping efficacy and pain acceptance; these studies have examined relationships between these factors and acute and chronic postsurgical pain. Studies of acute postsurgical pain in youth consistently support the association between higher levels of pain catastrophizing and pain anxiety and higher ratings of subsequent acute postoperative pain intensity. ${ }^{3,56,57}$ However, these studies assessed only acute pain; thus, it is unclear whether there is an association with longer term pain outcomes. Only one study found a relationship between pain-related anxiety, measured one week after surgery, and subsequent CPSP. ${ }^{11}$ The majority of studies found no association between pain-related anxiety or pain catastrophizing, measured before surgery, and CPSP, ${ }^{10,13,16}$ pain vigilance and fear of movement were also unrelated to persistent pain. ${ }^{10,11}$ In contrast, in one study including resiliency factors (eg, pain coping efficacy before surgery), a relationship was found between higher resiliency and more rapid pain recovery over six months following surgery. ${ }^{26}$

Katz and Seltzer ${ }^{17}$ proposed that different psychological processes may underlie the transition from acute to chronic pain: while some factors may play a role in initial transition to chronicity after an injury (ie, initiating factors), other factors may be more salient for the maintenance and impact of established chronic pain (ie, maintaining factors). ${ }^{16}$ Pediatric studies point to a more consistent relationship between pain-related psychological factors and acute postsurgical pain, whereas more general psychological constructs were associated with CPSP. However, distinguishing these processes necessitates repeated assessment of psychological factors before and after surgery, as well as measurement of both acute and chronic postsurgical pain. Overall, future pediatric studies 
are needed using research designs that allow for examining temporal pathways of psychological processes (risk and resiliency factors) involved in acute to chronic pain transition.

\section{Behavioral Factors}

Recent research has investigated the role of behavioral factors, such as functional disability and sleep, on the development of CPSP. One study identified the level of functional disability before surgery as a predictor of CPSP. $^{10}$ Sleep has also emerged as an important risk factor, with longitudinal research finding that insufficient sleep before surgery places youth at risk for more severe acute pain and higher incidence of CPSP. ${ }^{16,57}$ Sleep after surgery continues to play a role in recovery: poor subjective sleep quality during the weeks and months following surgery was associated with higher chronic pain intensity. ${ }^{58}$ Further research applying objective sleep monitoring during the months following surgery and examining underlying neurobiological mechanisms is needed to gain understanding of how sleep-pain relationships evolve over time and influence acute to chronic pain transition.

\section{Parent and Family Factors}

Limited studies have examined the impact of parent and family factors on the incidence of pediatric CPSP. Two studies found a relationship between a specific parent factor (parental pain catastrophizing) and CPSP. ${ }^{13,59}$ However, more recent studies found that CPSP was not associated with parental pain anxiety, pain catastrophizing, pain flexibility, anxiety sensitivity, or depression, or with overall family functioning. ${ }^{10,16,38}$ In contrast, parent factors have been consistently related to outcomes associated with other types of pediatric chronic pain. ${ }^{60}$ In general, more research is needed to explore the relationships between a range of parent and family factors and pediatric CPSP. In particular, future research should examine parent behaviors and emotional functioning, the parent-child dyadic relationship, and global family functioning at baseline and over time to better understand possible influences on CPSP. ${ }^{61-64}$

Overall, the literature relating psychosocial factors to the development of CPSP in children and adolescents is inconsistent. Although there have been a fair number of studies focused on these relationships, studies differ in how and when psychosocial variables are measured, as well as in which psychosocial constructs (eg, inclusion of parent variables) are included in multivariate models.
Notably, psychosocial variables have largely been measured at isolated timepoints before or after surgery using retrospective measures. However, one study that combined multiple general and pain-specific psychological and behavioral risk factors (pain catastrophizing, pain interference, depression, fatigue) to identify a "high symptom cluster" of adolescents preparing for surgery found that this subgroup was more likely to develop chronic pain. ${ }^{51}$ This demonstrates that despite inconsistencies in the literature, it is likely that overall, psychosocial distress puts youth at elevated risk for CPSP.

\section{Sensory Processing}

Surgery produces an injury, which is processed on a sensory level by nociceptors that detect tissue or nerve damage and transmit this sensory information via the dorsal horn of the spinal cord. This information is subject to both amplification and modulation before reaching the cortical and subcortical regions of the brain. ${ }^{17,18,65}$ Limited pediatric perioperative studies have examined the influence of sensory processing on postoperative pain. One study examined the relationship between central nervous system catecholamines, which are known to be involved in descending pain modulation, ${ }^{66}$ and subacute postoperative pain, finding that elevated baseline levels of norepinephrine in cerebral spinal fluid and plasma were associated with higher pain intensity at six weeks. ${ }^{2}$ In adult studies, quantitative sensory testing (ie, application of standardized mechanical, thermal, and other sensory stimuli to quantify self-reported sensory perception) has been used to examine the relationship between pain modulation and subsequent acute and chronic pain. Specifically, inefficient conditioned pain modulation (CPM) was associated with greater morphine consumption and heightened pain sensitivity in the acute postoperative period, ${ }^{67}$ and with an increased rate of transitioning to chronic pain after major surgeries. ${ }^{68-70}$ Postsurgical literature in adults also highlights the importance of the peripheral nervous system in the development of postoperative pain, demonstrating associations between peripheral sensitization and CPSP. ${ }^{71-73}$ To our knowledge, no published studies have applied quantitative sensory testing to examine the relationship between sensory processing and the development of CPSP in youth. However, these studies are needed to better understand the possible role of sensory processing in the transition from acute to chronic pain in this population. 


\section{Biological Factors}

\section{Inflammatory and Endocrine Response}

Studies exploring risk factors for CPSP in adults have emphasized the influence of the inflammatory response on postoperative pain. Immune cells and inflammatory molecules have been cited as a possible factors involved in the transition from acute to chronic postsurgical pain, ${ }^{74,75}$ and circulating markers such as the neutrophil to lymphocyte ratio and C-reactive protein have been associated with CPSP $^{76,77}$ in adults. In the acute postsurgical literature, a single available pediatric study did not find a relationship between preoperative salivary cortisol concentrations and acute postoperative pain. ${ }^{43}$ However, the role of inflammatory factors on CPSP has yet to be fully studied in pediatric surgery populations. The dearth of information regarding the effect of inflammatory and endocrine responses on pediatric CPSP highlights a novel avenue for investigation: future studies are needed to elucidate how these biological factors may be related to the development of CPSP in children.

\section{Epigenetic Factors}

In addition to the premorbid genetic profile, changes in gene function through DNA methylation, histone modification, and micro-RNA (miRNA) interference can occur in response to acute injury. For example, inflammation, nerve injury, drugs, and psychological stressors can induce changes in epigenetic processes that alter the expression of genes involved in immune/inflammatory, pain, and opioid receptor pathways, which in turn may influence the development of CPSP. ${ }^{29}$ This is an area of growing research interest, and these complex pathways have been extremely understudied in children.

\section{Future Directions}

Considerable gaps and opportunities exist for further investigations focused on mechanisms underlying the transition from acute to chronic postsurgical pain in children to inform prevention and intervention strategies. Future research must address limitations in the current literature. First and foremost, a focus on the presence of persistent pain, without consideration of severity or impact, makes the relevance of study findings unclear. It is imperative that future studies apply a unifying definition of CPSP which includes impact on quality of life, as set forth by the International Association for the Study of Pain, ${ }^{6,7}$ in order to allow comparison and pooling of data. Available validated measures of quality of life that have been used in the pediatric surgery population include the Pediatric Quality of Life Inventory (PedsQL) and the Scoliosis Research Society (SRS) measures. ${ }^{64}$ The inclusion of core acute and chronic pain outcomes highlighted in the Pediatric Initiative on Methods, Measurement, and Pain Assessment in Clinical Trials (PedIMMPACT) recommendations, such as physical, emotional, and role functioning, as well as sleep and economic factors, will also be critical to guide intervention research focused on improving outcomes in this population. ${ }^{78}$ Furthermore, most studies measure risk factors and outcomes at isolated timepoints. In order to implicate biopsychosocial processes as potential mechanisms, it is necessary to show temporal relationships between changes in these processes and the development of chronic pain over time. Longitudinal assessment of risk factors and pain over the transition from acute to chronic postsurgical pain presents an important avenue of potential investigation. Ideally, such studies would conduct repeated assessments up to at least six months after surgery in order to fully capture the transition to CPSP.

Whereas most studies have focused on psychosocial risk factors for CPSP, there are several important gaps in the understanding of other biobehavioral factors. In particular, there are opportunities for future research to examine psychosocial resiliency factors and to apply quantitative sensory testing to examine how changes in sensory and psychosocial processes after surgery relate to the transition to chronic pain. Similarly, the relationships between sleep and pain during the transition from acute to chronic pain comprise another understudied area in the literature. Early studies have identified subjective sleep quality as a potential promotor of chronic pain, but it will be important to employ objective measures of sleep to gain deeper insights into the possible mechanistic role of sleep deficiency. Such research may inform interventions targeting sleep during recovery. In addition, biological factors, including inflammatory, endocrine, and epigenetic responses to surgery, present an opportunity for further mechanistic studies in pediatric populations. Finally, the inclusion of larger and more diverse samples is necessary to understand the impact of social determinants of health on the transition to chronic pain.

While pediatric studies focusing on CPSP are expanding, further application of this model of acute to chronic pain transition to non-surgical injury populations, such as pediatric trauma and burns, could further expand our 
understanding of the development of chronic pain in other contexts.

Future research is also needed to test intervention strategies stemming from our conceptual model of biopsychosocial mechanisms of transition from acute to chronic postsurgical pain. A range of behavioral and pharmacological intervention strategies that target currently identified mechanisms and risk factors, such as sleep deficiency, psychosocial distress, and acute pain and recovery, could be developed and studied. The use of conceptual models to guide intervention approaches will significantly advance this field.

\section{Conclusion}

CPSP is a common and impactful condition affecting youth after surgery. As pain transitions from acute to chronic, alterations in sensory pain processing and in psychosocial processes may promote the evolution of chronic pain, while premorbid and injury-related factors may influence this process. Longitudinal studies are needed to elucidate key psychosocial and sensory mechanisms underlying risk for chronic pain. Studies examining response to surgery, both in the peripheral and central nervous systems and in other biological systems, will be critical to understanding how changes in these systems may promote the transition from acute to chronic pain. Future research is critically needed to test intervention strategies stemming from this conceptual model of biopsychosocial mechanisms of transition from acute to chronic postsurgical pain.

\section{Funding}

This work was supported by the National Institute of Arthritis, Musculoskeletal, and Skin Diseases under Award No. R01AR073780 (Principal Investigator: Rabbitts, J.A.).

\section{Disclosure}

The authors report no conflicts of interest for this work.

\section{References}

1. Groenewald CB, Rabbitts JA, Schroeder DR, Harrison TE. Prevalence of moderate-severe pain in hospitalized children. Paediatr Anaesth. 2012;22(7):661-668. doi:10.1111/j.1460-9592.2012.03807.x

2. Ferland CE, Parent AJ, Saran N, et al. Preoperative norepinephrine levels in cerebrospinal fluid and plasma correlate with pain intensity after pediatric spine surgery. Spine Deformity. 2017;5(5):325-333. doi:10.1016/j.jspd.2017.03.012

3. Page MG, Stinson S, Campbell C, Isaac I, Katz J. Pain-related psychological correlates of pediatric acute post-surgical pain. Journal of Pain Research. 2012;5:547-558. doi:10.2147/JPR.S36614
4. Rabbitts JA, Groenewald CB, Zhou C. Subacute Pain Trajectories following major musculoskeletal surgery in adolescents: A Pilot Study. Can J Pain. 2020;in press. doi:10.1080/ 24740527.2020.1765692

5. Rabbitts JA, Palermo TM, Zhou C, Pain M-SR. Health-related quality of life after pediatric inpatient surgery. J Pain. 2015;16 (12):1334-1341.

6. Williams G, Howard RF, Liossi C. Persistent postsurgical pain in children and young people: prediction, prevention, and management. Pain Rep. 2017;2(5):e616.

7. Werner MU, Kongsgaard UEI. Defining persistent post-surgical pain: is an update required? Br J Anaesth. 2014;113(1):1-4.

8. Mossetti V, Boretsky K, Astuto M, et al. Persistent pain following common outpatient surgeries in children: A multicenter study in Italy. Paediatr Anaesth. 2018;28(3):231-236.

9. Batoz H, Semjen F, Bordes-Demolis M, Benard A, Nouette-Gaulain $\mathrm{K}$. Chronic postsurgical pain in children: prevalence and risk factors. A prospective observational study. $B r \quad J$ Anaesth. 2016;117 (4):489-496.

10. Rosenbloom BN, Page MG, Isaac L, et al. Pediatric Chronic Postsurgical Pain And Functional Disability: A Prospective Study Of Risk Factors Up To One Year After Major Surgery. Journal of Pain Research. 2019;12:3079-3098. doi:10.2147/JPR.S210594

11. Horn-Hofmann C, Scheel J, Dimova V, et al. Prediction of persistent post-operative pain: pain-specific psychological variables compared with acute post-operative pain and general psychological variables. Eur J Pain. 2018;22(1):191-202. doi:10.1002/ejp.1115

12. Sieberg CB, Simons LE, Edelstein MR, et al. Pain prevalence and trajectories following pediatric spinal fusion surgery. J Pain. 2013;14 (12):1694-1702. doi:10.1016/j.jpain.2013.09.005

13. Rabbitts JA, Zhou C, Groenewald CB, Durkin L, Palermo TM. Trajectories of postsurgical pain in children: risk factors and impact of late pain recovery on long-term health outcomes after major surgery. Pain. 2015;156(11):2383-2389. doi:10.1097/j.pain.0000000 000000281

14. Groenewald CB, Essner BS, Wright D, Fesinmeyer MD, Palermo TM. The economic costs of chronic pain among a cohort of treatment-seeking adolescents in the United States. J Pain. 2014;15(9):925-933. doi:10.1016/j.jpain.2014.06.002

15. Datz H, Tumin D, Miller R, Smith TP, Bhalla T, Tobias JD. Pediatric chronic pain and caregiver burden in a national survey. Scand J Pain. 2019;19(1):109-116. doi:10.1515/sjpain-2018-0121

16. Rabbitts JA, Palermo TM, Zhou C, Meyyappan A, Chen L. Psychosocial predictors of acute and chronic pain in adolescents undergoing major musculoskeletal surgery. $J$ Pain;2020. doi:10.1016/j.jpain.2020.02.004

17. Katz J, Seltzer Z. Transition from acute to chronic postsurgical pain: risk factors and protective factors. Expert Rev Neurother. 2009;9 (5):723-744. doi:10.1586/ern.09.20

18. Elman I, Borsook D. Common Brain Mechanisms of Chronic Pain and Addiction. Neuron. 2016;89(1):11-36. doi:10.1016/j.neuron. 2015.11.027

19. Simons LE, Kaczynski KJ. The Fear Avoidance model of chronic pain: examination for pediatric application. J Pain. 2012;13 (9):827-835. doi:10.1016/j.jpain.2012.05.002

20. Kotzer AM. Factors predicting postoperative pain in children and adolescents following spine fusion. Issues in Comprehensive Pediatric Nursing. 2000;23(2):83-102. doi:10.1080/01460860050121411

21. Palermo TM, Drotar D. Prediction of Children's Postoperative Pain: the Role of Presurgical Expectations and Anticipatory Emotions. Journal of Pediatric Psychology. 1996;21(5):683-698. doi:10.1093/ jpepsy/21.5.683

22. Schmitz A-K, Vierhaus M, Lohaus A. Pain tolerance in children and adolescents: sex differences and psychosocial influences on pain threshold and endurance. Eur J Pain. 2013;17(1):124-131. doi:10.10 02/j.1532-2149.2012.00169.x 
23. Gobina I, Villberg J, Valimaa R, et al. Prevalence of self-reported chronic pain among adolescents: evidence from 42 countries and regions. Eur J Pain. 2019;23(2):316-326. doi:10.1002/ejp.1306

24. Huguet A, Tougas ME, Hayden J, et al. Systematic Review of Childhood and Adolescent Risk and Prognostic Factors for Recurrent Headaches. J Pain. 2016;17(8):855-873e858. doi:10. 1016/j.jpain.2016.03.010

25. Vierhaus M, Lohaus A, Schmitz AK. Sex, gender, coping, and self-efficacy: mediation of sex differences in pain perception in children and adolescents. Eur J Pain. 2011;15(6):621e621.

26. Connelly M, Fulmer RD, Prohaska J, et al. Predictors of postoperative pain trajectories in adolescent idiopathic scoliosis. Spine. 2014;39(3):E174-181. doi:10.1097/BRS.0000000000000099

27. Hwang SW, Pendleton C, Samdani AF, et al. Preoperative SRS pain score is the primary predictor of postoperative pain after surgery for adolescent idiopathic scoliosis: an observational retrospective study of pain outcomes from a registry of 1744 patients with a mean follow-up of 3.4 years. Eur Spine J. 2020;29(4):754-760. doi:10.1007/s00586-020-06293-y

28. Page MG, Stinson S, Campbell C, Isaac I, Katz J. Identification of pain-related psychological risk factors for the development and maintenance of pediatric chronic postsurgical pain. Journal of Pain Research. 2013;6:167-180. doi:10.2147/JPR.S40846

29. James SK. Chronic postsurgical pain: is there a possible genetic link? Br J Pain. 2017;11(4):178-185. doi:10.1177/2049463717723222

30. Zubieta J. COMT val158met Genotype Affects micro-Opioid Neurotransmitter Responses to a Pain Stressor. Science. 2003;299 (5610):1240-1243. doi:10.1126/science. 1078546

31. Diatchenko L, Slade GD, Nackley AG, et al. Genetic basis for individual variations in pain perception and the development of a chronic pain condition. Hum Mol Genet. 2005;14(1):135-143. doi:10.1093/hmg/ddi013

32. Kehlet H, Jensen TS, Woolf CJ. Persistent postsurgical pain: risk factors and prevention. Lancet. 2006;367(9522):1618-1625. doi:10.1016/S0140-6736(06)68700-X

33. Hoofwijk DM, van Reij RR, Rutten BP, Kenis G, Buhre WF, Joosten EA. Genetic polymorphisms and their association with the prevalence and severity of chronic postsurgical pain: a systematic review. Br J Anaesth. 2016;117(6):708-719. doi:10.1093/bja/aew378

34. Hoofwijk DMN, van Reij RRI, Rutten BPF, et al. Genetic polymorphisms and prediction of chronic post-surgical pain after hysterectomy - a subgroup analysis of a multicenter cohort study. Acta Anaesthesiol Scand. 2019;63(8):1063-1073. doi:10.1111/aas.13413

35. Sadhasivam S, Chidambaran V, Olbrecht VA, et al. Genetics of pain perception, COMT and postoperative pain management in children. Pharmacogenomics. 2014;15(3):277-284.

36. Chidambaran V, Zhang X, Martin LJ, et al. DNA methylation at the mu-1 opioid receptor gene (OPRM1) promoter predicts preoperative, acute, and chronic postsurgical pain after spine fusion. Pharmgenomics Pers Med. 2017;10:157-168.

37. Chidambaran V, Zhang X, Geisler K, et al. Enrichment of Genomic Pathways Based on Differential DNA Methylation Associated With Chronic Postsurgical Pain and Anxiety in Children: A Prospective, Pilot Study. J Pain. 2019;20(7):771-785.

38. Chidambaran V, Ding L, Moore DL, et al. Predicting the pain continuum after adolescent idiopathic scoliosis surgery: A prospective cohort study. Eur J Pain. 2017;21(7):1252-1265.

39. Chidambaran V, Gang Y, Pilipenko V, Ashton M, Ding L. Systematic Review and Meta-Analysis of Genetic Risk of Developing Chronic Postsurgical Pain. J Pain. 2019.

40. Langford DJ, Paul SM, West CM, et al. Variations in potassium channel genes are associated with distinct trajectories of persistent breast pain after breast cancer surgery. Pain. 2015;156(3):371-380.

41. Costigan M, Belfer I, Griffin RS, et al. Multiple chronic pain states are associated with a common amino acid-changing allele in KCNS1. Brain. 2010;133(9):2519-2527.
42. Clarke H, Katz J, Flor H, Rietschel M, Diehl SR, Seltzer Z. Genetics of chronic post-surgical pain: a crucial step toward personal pain medicine. Can J Anaesth. 2015;62(3):294-303.

43. Ferland CE, Saran N, Valois T, et al. Preoperative Distress Factors Predicting Postoperative Pain in Adolescents Undergoing Surgery: A Preliminary Study. J Pediatr Health Care. 2017;31(1):5-15.

44. Ocay DD, Li MMJ, Ingelmo P, Ouellet JA, Page MG, Ferland CE. Predicting Acute Postoperative Pain Trajectories and Long-Term Outcomes of Adolescents after Spinal Fusion Surgery. Pain Res Manag. 2020;2020:9874739.

45. Bastrom TP, Marks MC, Yaszay B, Newton PO, Harms Study G. Prevalence of postoperative pain in adolescent idiopathic scoliosis and the association with preoperative pain. Spine. 2013;38 (21):1848-1852.

46. Julien-Marsollier F, David R, Hilly J, Brasher C, Michelet D, Dahmani S. Predictors of chronic neuropathic pain after scoliosis surgery in children. Scand J Pain. 2017;17:339-344.

47. Brummett CM, Janda AM, Schueller CM, et al. Survey criteria for fibromyalgia independently predict increased postoperative opioid consumption after lower-extremity joint arthroplasty: a prospective, observational cohort study. Anesthesiology. 2013;119(6):1434-1443.

48. Brummett CM, Urquhart AG, Hassett AL, et al. Characteristics of fibromyalgia independently predict poorer long-term analgesic outcomes following total knee and hip arthroplasty. Arthritis Rheumatol. 2015;67(5):1386-1394.

49. Hilliard PE, Waljee J, Moser S, et al. Prevalence of Preoperative Opioid Use and Characteristics Associated With Opioid Use Among Patients Presenting for Surgery. JAMA Surg. 2018;153(10):929-937.

50. Janda AM, As-Sanie S, Rajala B, et al. Fibromyalgia survey criteria are associated with increased postoperative opioid consumption in women undergoing hysterectomy. Anesthesiology. 2015;122 (5):1103-1111.

51. Voepel-Lewis T, Caird MS, Tait AR, et al. A cluster of high psychological and somatic symptoms in children with idiopathic scoliosis predicts persistent pain and analgesic use 1 year after spine fusion. Paediatr Anaesth. 2018;28(10):873-880.

52. Voepel-Lewis T, Caird MS, Tait AR, et al. A High Preoperative Pain and Symptom Profile Predicts Worse Pain Outcomes for Children After Spine Fusion Surgery. Anesth Analg. 2017;124(5):1594-1602.

53. Landman Z, Oswald T, Sanders J, Diab M. Spinal Deformity Study G. Prevalence and predictors of pain in surgical treatment of adolescent idiopathic scoliosis. Spine. 2011;36(10):825-829.

54. Li MM, Ocay DD, Teles AR, et al. Acute postoperative opioid consumption trajectories and long-term outcomes in pediatric patients after spine surgery. J Pain Res. 2019;12:1673-1684.

55. Edwards RR, Dworkin RH, Sullivan MD, Turk DC, Wasan AD. The Role of Psychosocial Processes in the Development and Maintenance of Chronic Pain. J Pain. 2016;17(9 Suppl):T70-92.

56. Esteve R, Marquina-Aponte V, Ramirez-Maestre C. Postoperative pain in children: association between anxiety sensitivity, pain catastrophizing, and female caregivers' responses to children's pain. $J$ Pain. 2014;15(2):157-168 e151.

57. Rabbitts JA, Groenewald CB, Tai GG, Palermo TM. Presurgical psychosocial predictors of acute postsurgical pain and quality of life in children undergoing major surgery. $J$ Pain. 2015;16 (3):226-234

58. Rabbitts JA, Zhou C, Narayanan A, Palermo TM. Longitudinal and Temporal Associations Between Daily Pain and Sleep Patterns After Major Pediatric Surgery. J Pain. 2017;18(6):656-663.

59. Page MG, Campbell F, Isaac L, Stinson J, Katz J. Parental risk factors for the development of pediatric acute and chronic postsurgical pain: a longitudinal study. J Pain Res. 2013;6:727-741.

60. Donnelly TJ, Palermo TM, Newton-John TRO. Parent cognitive, behavioural, and affective factors and their relation to child pain and functioning in pediatric chronic pain: a systematic review and meta-analysis. Pain. 2020. 
61. Birnie KA, Chorney J, El-Hawary R, Group PS. Child and parent pain catastrophizing and pain from presurgery to 6 weeks postsurgery: examination of cross-sectional and longitudinal actor-partner effects. Pain. 2017;158(10):1886-1892.

62. Noel M, Rabbitts JA, Fales J, Chorney J, Palermo TM. The influence of pain memories on children's and adolescents' post-surgical pain experience: A longitudinal dyadic analysis. Health Psychol. 2017;36 (10):987-995.

63. Rabbitts JA, Fisher E. Postsurgical pain in children: unraveling the interplay between child and parent psychosocial factors. Pain. 2017;158(10):1847-1848.

64. Rabbitts JA, Fisher E, Rosenbloom BN, Palermo TM. Prevalence and Predictors of Chronic Postsurgical Pain in Children: A Systematic Review and Meta-Analysis. J Pain. 2017;18(6):605-614.

65. Tracey I. Getting the pain you expect: mechanisms of placebo, nocebo and reappraisal effects in humans. Nat Med. 2010;16 (11):1277-1283.

66. D'Mello R, Dickenson AH. Spinal cord mechanisms of pain. $\mathrm{Br}$ J Anaesth. 2008;101(1):8-16.

67. Grosen K, Vase L, Pilegaard HK, Pfeiffer-Jensen M, Drewes AM. Conditioned pain modulation and situational pain catastrophizing as preoperative predictors of pain following chest wall surgery: a prospective observational cohort study. PLoS One. 2014;9(2):e90185.

68. Yarnitsky D, Crispel Y, Eisenberg E, et al. Prediction of chronic post-operative pain: pre-operative DNIC testing identifies patients at risk. Pain. 2008;138(1):22-28.

69. Wilder-Smith OH, Schreyer T, Scheffer GJ, Arendt-Nielsen L. Patients with chronic pain after abdominal surgery show less preoperative endogenous pain inhibition and more postoperative hyperalgesia: a pilot study. J Pain Palliat Care Pharmacother. 2010;24 (2):119-128.
70. Landau R, Kraft JC, Flint LY, et al. An experimental paradigm for the prediction of Post-Operative Pain (PPOP. J Vis Exp. 2010;35.

71. Kim DH, Pearson-Chauhan KM, McCarthy RJ, Buvanendran A. Predictive Factors for Developing Chronic Pain After Total Knee Arthroplasty. J Arthroplasty. 2018;33(11):3372-3378.

72. Wylde V, Palmer S, Learmonth ID, Dieppe P. The association between pre-operative pain sensitisation and chronic pain after knee replacement: an exploratory study. Osteoarthritis Cartilage. 2013;21 (9):1253-1256.

73. Wright A, Moss P, Sloan K, et al. Abnormal quantitative sensory testing is associated with persistent pain one year after TKA. Clin Orthop Relat Res. 2015;473(1):246-254

74. Voscopoulos C, Lema M. When does acute pain become chronic? $\mathrm{Br}$ J Anaesth. 2010;105(Suppl 1):i69-85.

75. Richebe P, Capdevila X, Rivat C. Persistent Postsurgical Pain: pathophysiology and Preventative Pharmacologic Considerations. Anesthesiology. 2018;129(3):590-607.

76. Bugada D, Lavand'homme P, Ambrosoli AL, et al. Effect of Preoperative Inflammatory Status and Comorbidities on Pain Resolution and Persistent Postsurgical Pain after Inguinal Hernia Repair. Mediators Inflamm. 2016;2016:5830347.

77. Hashimoto K, Tsuji A, Takenaka S, et al. C-reactive Protein Level on Postoperative Day One is Associated with Chronic Postsurgical Pain After Mastectomy. Anesth Pain Med. 2018;8(4):e79331.

78. McGrath PJ, Walco GA, Turk DC, et al. Core outcome domains and measures for pediatric acute and chronic/recurrent pain clinical trials: pedIMMPACT recommendations. J Pain. 2008;9(9):771-783.

\section{Publish your work in this journal}

The Journal of Pain Research is an international, peer reviewed, open access, online journal that welcomes laboratory and clinical findings in the fields of pain research and the prevention and management of pain. Original research, reviews, symposium reports, hypothesis formation and commentaries are all considered for publication. The manuscript management system is completely online and includes a very quick and fair peer-review system, which is all easy to use. Visit http:// www.dovepress.com/testimonials.php to read real quotes from published authors. 